\title{
Aesthetic Rehabilitation of a Patient with Amelogenesis Imperfecta - A Case Report
}

\author{
Rajesh Shetty ${ }^{1}$, Sanath Kumar Shetty², Naresh Shetty ${ }^{3}$, Shriya Deepak Jain ${ }^{4}$, Karkala Syed Suhaim ${ }^{5}$ \\ 1, 2, 3, 4, 5 Department of Prosthodontics, Yenepoya Dental College, Deralakatte, Mangalore, Karnataka, India.
}

\section{INTRODUCTION}

Amelogenesis imperfecta (AI) is a developmental disorder that affects the internal and external morphology of the enamel thereby altering the structure as well as the clinical appearance of the tooth. ${ }^{1}$ It is usually not associated with any systemic disorder. AI results mainly due to the improper differentiation of ameloblasts, resulting in incomplete or complete absence of enamel. ${ }^{2}$

AI can affect both primary and the permanent dentition. The common manifestations of amelogenesis imperfecta ranges from discoloration of tooth (yellow, brown, or grey), areas of dentine exposure leading to hypersensitivity, pitted enamel leading to increased plaque accumulation causing increased incidence of caries and periodontal problems, taurodontism, impacted permanent teeth; and anterior or posterior open bite malocclusions. ${ }^{3.4}$

Three types of amelogenesis imperfecta can be diagnosed depending on the clinical and histological characteristics, whereas radiological interpretation shows normal dental pulp and root morphology in all the three types. ${ }^{5}$

AI type I (hypoplastic) the enamel presents with normal density and calcification characteristics. This type is also characterised by deficient quantity of enamel. The enamel appears hard but is malformed. 5

AI type II (hypomature) is attributed to abnormalities in the maturation stage of enamel formation which results in a mottled appearance, opaque white to red-brown coloration. The thickness of enamel in this type is more regular than type I. The clinical features of this type of AI are characterised by normal thickness of enamel, which flakes very easily and is liable to abrasion. ${ }^{5,6}$

AI type III (hypocalcified) is characterised by a defect in the enamel calcification. The enamel formed is of normal thickness, but is poorly mineralised. The structure of enamel is weak and the teeth appear chalky and opaque in appearance. Radiographically, the enamel appears less radio opaque when compared to dentine. ${ }^{1}$

This case report deals with the best suitable aesthetic management in a clinical scenario of a young adult diagnosed with type I amelogenesis imperfecta. Aesthetic rehabilitation was done on anterior teeth of maxillary and mandibular teeth with porcelain laminate veneers.
Corresponding Author: Dr. Shriya Deepak Jain, C Block, Gardeynia Hostel, Yenepoya University, Mangalore - 575018,

Karnataka, India.

E-mail: shriyajjain@gmail.com

DOI: $10.14260 /$ jemds/2021/89

How to Cite This Article:

Shetty $R$, Shetty SK, Shetty N, et al. Aesthetic rehabilitation of a patient with amelogenesis imperfect - a case report. $J$ Evolution Med Dent Sci 2021;10(06):401403, DOI: 10.14260/jemds/2021/89

Submission 05-10-2020,

Peer Review 12-12-2020,

Acceptance 17-12-2020,

Published 08-02-2021.

Copyright @ 2021 Rajesh Shetty et al. This is an open access article distributed under Creative Commons Attribution License [Attribution 4.0 International (CC BY 4.0)] 


\section{PRESENTATION OF CASE}

A 21-year-old female patient reported to the Department of Prosthodontics, Yenepoya Dental College, Mangalore with a chief complaint of poor aesthetics in anterior and posterior regions of upper and lower teeth and desired for aesthetic correction of the same. After detailed patient examination the condition was diagnosed as type 1 amelogenesis imperfecta. (Figure 1)

\section{DISCUSSION OF MANAGEMENT}

A diagnostic radiograph was taken to rule out any pulpal involvement and periapical pathologies. 37,47 had carious lesions extending beyond the pulp. All other teeth appeared intact in the radiographs.

Impressions of both the arches was made with alginate (Zhermack) in a stock tray, facebow transfer was recorded and diagnostic mounting was done on a semi adjustable articulator.

Porcelain laminate veneers were planned for the anterior teeth as it allows for minimum tooth preparation and thereby reduces chances of post-operative sensitivity and full coverage porcelain-fused-to-metal (PFM) restorations were planned for the decayed posterior teeth. As the patient's primary concern was aesthetics, patient postponed the treatment for posterior teeth and only wanted aesthetic correction with regards to maxillary and mandibular anterior teeth.

The facial aspect of $13,12,11,21,22,23$ was prepared first. Window preparation was done and a total of $0.5 \mathrm{~mm}$ of tooth preparation was done. Self-limiting depth-cutting disks of 0.5 mm thickness was used to make the initial depth cuts, and 1.4 $\mathrm{mm}$ chamfer diamond point was used later to merge the depth cuts created. A chamfer finish line was prepared. The preparation was devoid of any sharp line angles or any undercuts (Figure 2). As the incisal edges and cuspal tips were not prepared, occlusal factors did not play a major role in this rehabilitation.

Impression was made using polyvinylsiloxane (Zhermack) using double step double mix technique. The prepared teeth were temporarily restored with composite material (3M ESPE Filtek Z350 XT composite syringe) following spot etching for 20 seconds. The same clinical and laboratory steps were followed for the mandibular anterior teeth i.r.t 31, 32, 33, 41, 42 and 43.

The porcelain laminate veneers were fabricated with lithium disilicate (IPS e-max Press) for maximum aesthetics (Figure 3, 4, 5, 6). The internal surface of the laminates was etched with $5 \%$ hydrofluoric acid. The temporary restorations were removed with utmost care. The residual composite cement was removed with probe and polishing points. The laminates were tried to verify the fit and aesthetics. The intaglio surface of the laminates was treated with silane coupling agent (Monobond-S-Single-Component Silane Coupling Agent). Cheeks were retracted and cotton rolls were placed to achieve a moisture free environment. The teeth were acid etched (3M Espe Scotchbond Etchant) for 20 seconds and then vigorously washed with water till the etchant was completely removed.
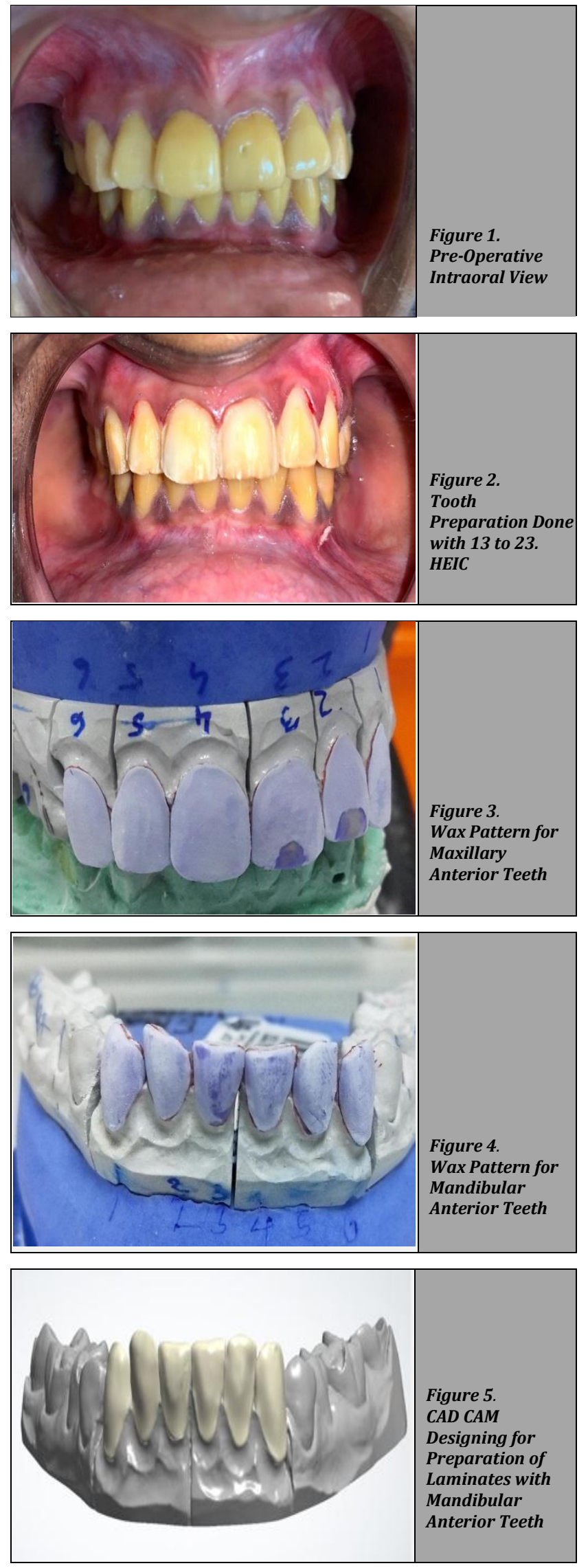

This was followed by application of universal bonding agent (3M Single bond universal adhesive) and light cured for 20 seconds. After bonding, a light cure veneer luting cement (3M Espe RelyX ${ }^{\mathrm{TM}}$ Veneer Cement) was applied on the under surface of the laminates and was placed on the teeth starting 
with canine of one side to the other. It was tack cured for 2 seconds, and the excess cement material was removed with hand instruments and dental floss. The laminates were then cured for 60 seconds (Figure 7). After complete polymerisation, post-operative instructions were given to the patient. The patient was satisfied with the aesthetics and was recalled after one week for regular check-up.
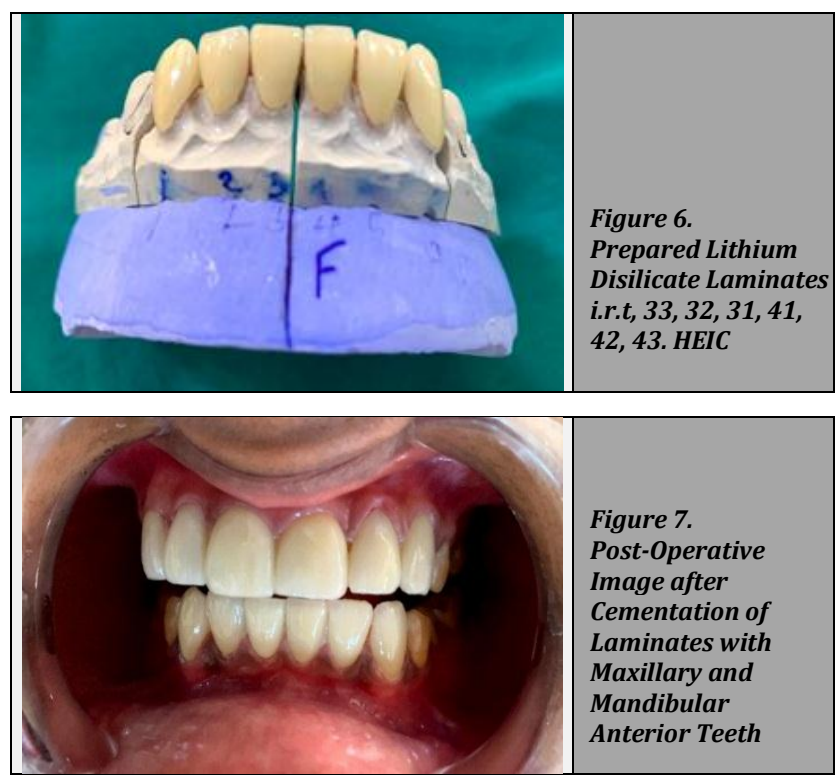

\section{DISCUSSION}

There are various treatment modalities that can be preferred for a patient with amelogenesis imperfecta. There have been a number of case reports in literature indicating the use of full coverage restorations for such clinical scenarios, however this is usually associated with huge amount of loss of tooth structure. ${ }^{1}$ As the patient was young, and also to follow the conservative approach of treatment, porcelain laminate veneers were planned for the patient.

In the present case report, aesthetic rehabilitation was done on anterior maxillary and mandibular teeth with the porcelain laminate veneers which improved patient's aesthetics. The treatment modality opted for this case was porcelain laminates and veneers, as it involves minimum tooth reduction.

Financial or other competing interests: None.

Disclosure forms provided by the authors are available with the full text of this article at jemds.com.

\section{REFERENCES}

[1] Gadhia K, McDonald S, Arkutu N, et al. Amelogenesis imperfecta: an introduction. Br Dent J 2012;212(8):3779.

[2] Seymen F, Kiziltan B. Amelogenesis imperfecta: a scanning electron microscopic and histopathologic study. J Clin Pediatric Dent 2002;26(4):327-35.

[3] Gokce K, Canpolat C, Ozel E. Restoring function and esthetics in a patient with amelogenesis imperfecta: a case report. J Contemp Dent Pract 2007;8(4):95-101.

[4] Yip HK, Smales RJ. Oral rehabilitation of young adults with amelogenesis imperfecta. Int $\mathrm{J}$ Prosthodont 2003;16(4):345-9.

[5] Luzzi V, Bossù $M$, Cavallè $E$, et al. Case report: clinical management of hypoplastic amelogenesis imperfecta. Eur J Paediatr Dent 2003;4(3):149-54.

[6] Akin H, Tasveren S, Yeler DY. Interdisciplinary approach to treating a patient with amelogenesis imperfecta: a clinical report. J Esthet Restor Dent 2007;19(3):131-5. 